\title{
CTRP12 ameliorates atherosclerosis by promoting cholesterol efflux and inhibiting inflammatory response via the miR-155-5p/LXRa pathway
}

\author{
Gang Wang ${ }^{1}$, Jiao-Jiao Chen ${ }^{2}$, Wen-Yi Deng ${ }^{2}$, Kun Ren ${ }^{2,3}$, Shan-Hui Yin ${ }^{4}$ and Xiao-Hua Yu ${ }^{2}$
}

\begin{abstract}
C1q tumor necrosis factor-related protein 12 (CTRP12), a conserved paralog of adiponectin, is closely associated with cardiovascular disease. However, little is known about its role in atherogenesis. The aim of this study was to examine the influence of CTRP12 on atherosclerosis and explore the underlying mechanisms. Our results showed that lentivirus-mediated CTRP12 overexpression inhibited lipid accumulation and inflammatory response in lipid-laden macrophages. Mechanistically, CTRP12 decreased miR-155-5p levels and then increased its target gene liver X receptor a (LXRa) expression, which increased ATP binding cassette transporter A1 (ABCA1)- and ABCG1-dependent cholesterol efflux and promoted macrophage polarization to the M2 phenotype. Injection of lentiviral vector expressing CTRP12 decreased atherosclerotic lesion area, elevated plasma high-density lipoprotein cholesterol levels, promoted reverse cholesterol transport $(\mathrm{RCT})$, and alleviated inflammatory response in apolipoprotein E-deficient $\left(\mathrm{apoE}^{-1-}\right)$ mice fed a Western diet. Similar to the findings of in vitro experiments, CTRP12 overexpression diminished miR-155-5p levels but increased LXRa, ABCA1, and ABCG1 expression in the aortas of apoE ${ }^{-1-}$ mice. Taken together, these results suggest that CTRP12 protects against atherosclerosis by enhancing RCT efficiency and mitigating vascular inflammation via the miR-155-5p/LXRa pathway. Stimulating CTRP12 production could be a novel approach for reducing atherosclerosis.
\end{abstract}

\section{Introduction}

Atherosclerosis, the pathological basis of most cardiovascular disease, is a chronic vascular inflammatory disease characterized by dysregulated lipid homeostasis ${ }^{1}$. As a major cell type in the atherosclerotic lesions, macrophages play a central role in the occurrence and development of atherosclerosis. During atherogenesis, circulating monocytes transmigrate into the subintima where they differentiate into macrophages. After uptake of large amounts of modified lipoproteins, such as oxidized low-density lipoprotein (ox-LDL), macrophages are transformed into lipid-rich foam cells, a hallmark of early-

\footnotetext{
Correspondence: Shan-Hui Yin (yinshanhui@163.com) or

Xiao-Hua Yu (yxh167@qq.com)

'Department of Cardiology, The First Affiliated Hospital of University of South China, Hengyang 421001 Hunan, China

${ }^{2}$ Institute of Clinical Medicine, The Second Affiliated Hospital of Hainan Medical University, Haikou 570100 Hainan, China

Full list of author information is available at the end of the article

Edited by A. Finazzi-Agrò
}

stage atherosclerotic lesions ${ }^{2}$. As a transmembrane protein, the primary function of ATP binding cassette (ABC) transporter $\mathrm{A} 1$ (ABCA1) is to mediate cholesterol efflux to lipid-poor apolipoprotein A-I (apoA-I) for generation of nascent high-density lipoprotein (HDL) particles. ABCG1, an important member of the ABCG subfamily, promotes the efflux of intracellular cholesterol to HDL. Decreased cholesterol efflux mediated by ABCA1 and ABCG1 is regarded as a critical mechanism for macrophage foam cell formation ${ }^{3-5}$. Studies from our groups and others have revealed that increased ABCA1 and ABCG1 expression contributes to alleviation of lipid accumulation and atherosclerotic lesions in atherosclerosis-prone mice $^{6,7}$. Moreover, macrophage foam cells can secrete a variety of pro-inflammatory mediators, leading to further development of atherosclerosis ${ }^{8}$. Thus, a better understanding of the regulatory mechanisms for cholesterol efflux and vascular inflammation is important to develop novel therapeutic strategies for atherosclerosis.

\section{(c) The Author(s) 2021}

(c) (i) Open Access This article is licensed under a Creative Commons Attribution 4.0 International License, which permits use, sharing, adaptation, distribution and reproduction cc) in any medium or format, as long as you give appropriate credit to the original author(s) and the source, provide a link to the Creative Commons license, and indicate if changes were made. The images or other third party material in this article are included in the article's Creative Commons license, unless indicated otherwise in a credit line to the material. If material is not included in the article's Creative Commons license and your intended use is not permitted by statutory regulation or exceeds the permitted use, you will need to obtain permission directly from the copyright holder. To view a copy of this license, visit http://creativecommons.org/licenses/by/4.0/. 
ABCA1 and ABCG1 are regulated by a variety of bioactive molecules. Liver X receptor $\alpha$ (LXR $\alpha$, encoded by the NR1H3 gene), a nuclear hormone receptor, is thought to be the most important transcription factor to induce $\mathrm{ABCA} 1$ and $\mathrm{ABCG} 1$ expression ${ }^{9}$. We and others previously reported that administration of leonurine, kuwanon $\mathrm{G}$ or dihydromyricetin inhibits lipid accumulation in THP-1 macrophages and protects against atherosclerosis in mice by activating the LXR $\alpha-\mathrm{ABCA} 1 /$ ABCG1 signaling pathways ${ }^{10-12}$. In addition to its role in the regulation of lipid metabolism, activation of LXR $\alpha$ can suppress inflammatory signaling in macrophages ${ }^{13}$. MicroRNAs (miRNAs) are a class of small noncoding RNA molecules that can post-transcriptionally modulate the expression of their downstream target genes. There is accumulating evidence that dysregulation of miRNAs is associated with lipid metabolism disorder, inflammatory response, and atherosclerosis progres$\operatorname{sion}^{14,15}$. MiR-155-5p is located in a region called $\mathrm{B}$ cell integration cluster on human chromosome $21^{16}$. Plasma miR-155-5p levels are significantly elevated in rheumatoid arthritis patients with subclinical atherosclerosis as compared to healthy volunteers ${ }^{17}$. Increased levels of circulating miR-155-5p are also observed in patients with unstable coronary artery disease ${ }^{18}$. Importantly, there is a negative correlation between circulating adipocyte-derived extracellular vesicle miR-155-5p isolated from adolescent obesity subjects and cholesterol efflux capacity in THP-1 macrophages ${ }^{19}$. Knockdown of miR-155-5p inhibits pro-inflammatory cytokine production and ameliorates lipopolysaccharide (LPS)induced acute lung injury in mice ${ }^{20}$. These findings suggest that miR-155-5p plays a role in the regulation of lipid metabolism and inflammation. It is still unclear, however, whether miR-155-5p affects these two processes by reducing the activity of LXR $\alpha$.

C1q tumor necrosis factor-related protein 12 (CTRP12), also known as adipolin, is a newly discovered adipokine and a conserved paralog of adiponectin ${ }^{21}$. Overexpression of CTRP12 was shown to ameliorate cardiomyocyte injury induced by LPS ${ }^{22}$. Deletion of CTRP12 in mice aggravates neointimal thickening after vascular injury ${ }^{23}$. In addition, circulating CTRP12 levels are significantly decreased in patients with coronary artery disease and show an independent correlation with the risk of this disease ${ }^{24}$. These findings suggest that CTRP12 is associated with cardiovascular disease. However, its role in atherogenesis is still unclear. In the present study, we demonstrated for the first time that CTRP12 mitigates atherosclerosis by promoting ABCA1/ABCG1-dependent cholesterol efflux and inhibiting inflammatory response via the miR-155-5p/ LXR $\alpha$ signaling pathway, thereby providing a novel target for the prevention and treatment of atherosclerotic cardiovascular disease.

\section{Materials and methods}

\section{Mice, diet and treatment}

Forty-five male 8-week-old apoE ${ }^{-1-}$ mice on a C57BL/6 background were purchased from Changzhou Cavens Lab Animal Co., Ltd (Jiangsu, China), and housed under a $12 \mathrm{~h}$ light/dark cycle in sterilized filter-topped cages with free access to food and water with a constant humidity ( $55 \pm 5 \%)$ and temperature $\left(23 \pm 1{ }^{\circ} \mathrm{C}\right)$. These mice were randomly divided into control group, LV-NC group, and LV-CTRP12 group with 15 animals in each group. Mice were fed a Western diet (21\% fat, 0.3\% cholesterol; Research Diets) for 12 weeks and simultaneously injected with PBS, LV-NC, or LV-CTRP12 $\left(2 \times 10^{9} \mathrm{TU} / \mathrm{mL}\right)$ via the tail vein once every 3 weeks. At the end of the study, mice were sacrificed and the heart, aorta, and blood samples were collected. All surgeries were conducted under sodium pentobarbital anesthesia. The animal experiments were approved by the Institutional Animal Care and Use Committee of the Second Affiliated Hospital of Hainan Medical University.

\section{THP-1 cell culture, mouse peritoneal macrophage (MPM) isolation, and CTRP12 overexpression}

THP-1 monocytes (TIB-202, American Type Culture Collection) were cultured in RPMI 1640 medium (SigmaAldrich, St. Louis, MO, USA) supplemented with $10 \%$ fetal bovine serum (FBS, Sigma-Aldrich) and $1 \%$ penicillin-streptomycin (Beyotime, Shanghai, China) at $37^{\circ} \mathrm{C}$ in a humidified atmosphere of $5 \% \mathrm{CO}_{2}$. To induce the differentiation of monocytes into macrophages, cells were treated with $100 \mathrm{nM}$ phorbol 12-myristate 13-acetate (PMA, Sigma-Aldrich) for $24 \mathrm{~h}$. Then, macrophages were incubated with $50 \mu \mathrm{g} / \mathrm{mL}$ ox-LDL (Yiyuan biotechnology, Guangzhou, China) for $48 \mathrm{~h}$ to become foam cells. Lentiviral vector expressing CTRP12 (LV-CTRP12) and empty vector (LV-NC) were provided by Genechem (Shanghai, China). THP-1 macrophage-derived foam cells were transfected with LV-CTRP12 or LV-NC at a multiplicity of infection of 100 in the presence of $8 \mathrm{mg} / \mathrm{mL}$ of polybrene for $24 \mathrm{~h}$. Cells in control group were incubated with PBS only. Thereafter, cells were cultured in fresh RPMI 1640 medium containing 10\% FBS for $48 \mathrm{~h}$. Western blot was performed to assess transfection efficiency.

MPMs were extracted as previously described ${ }^{25}$. Briefly, mice ( $n=5$ in each group) were intraperitoneally injected with 3\% thioglycolate three days prior to sacrifice. MPMs were isolated from the mouse peritoneal cavity with PBS. After centrifugation $\left(300 \mathrm{rpm}, 5 \mathrm{~min}, 4^{\circ} \mathrm{C}\right)$, cells were cultured in RPMI 1640 medium containing 10\% FBS and $1 \%$ penicillin-streptomycin.

\section{Evaluation of intracellular lipid droplets by Oil red 0 staining}

After treatment with PBS, LV-NC or LV-CTRP12, THP-1 macrophage-derived foam cells were fixed in $4 \%$ 
paraformaldehyde for $5 \mathrm{~min}$ and rinsed several times in PBS. These cells were then stained with Oil Red O working solution for $5 \mathrm{~min}$ at $37^{\circ} \mathrm{C}$ in the dark, and destained with $60 \%$ isopropanol for $10 \mathrm{~s}$. The stained cells were photographed under an inverted microscope (Olympus B×50).

\section{Detection of intracellular cholesterol and triglyceride (TG) contents}

High-performance liquid chromatography was used to measure intracellular cholesterol amounts as previously described $^{26}$. Briefly, cells were washed in PBS and lysed by sonication on ice using an ultrasonic processor (Scientz, Zhejiang, China). Protein concentrations in cell lysate supernatants were determined using a BCA protein assay kit (Beyotime). The cell lysates were supplemented with isovolumetric $15 \% \mathrm{KOH}$ (diluted with $150 \mathrm{~g} / \mathrm{L}$ ethanol) and vortexed. Cellular lipids were then extracted with nhexane-isopropanol $(3: 2, \mathrm{~V} / \mathrm{V})$ and dissolved in isopropanol $(50 \mathrm{mg} / \mathrm{mL})$. Cholesterol standard calibration solution ranging from 0 to $50 \mathrm{mg} / \mathrm{mL}$ was prepared. The reaction mixture (Tris- $\mathrm{HCl}(500 \mathrm{mM}, \mathrm{pH}=7.4), \mathrm{MgCl}_{2}$ $(500 \mathrm{mM})$, dithiothreitol $(10 \mathrm{mM})$ and $5 \% \mathrm{NaCl})$, was added into $0.1 \mathrm{~mL}$ of cell solution or cholesterol standard calibration solution. The amount of total cholesterol (TC) was detected by adding $0.4 \mathrm{U}$ cholesterol oxidase combined with $0.4 \mathrm{U}$ cholesterol esterase, and free cholesterol (FC) content was determined by supplementing $0.4 \mathrm{U}$ cholesterol oxidase alone. Each reaction tube was incubated at $37^{\circ} \mathrm{C}$ for $30 \mathrm{~min}$, and the reaction was terminated by adding $100 \mu \mathrm{L}$ of ethanol: methanol $(1: 1 \mathrm{~V} / \mathrm{V})$. After centrifugation, the supernatants were collected and analyzed using 2790 Chromatographer (Waters, MA, USA). Absorbance at $216 \mathrm{~nm}$ was monitored. Data were analyzed by the TotalChrom software (PerkinElmer, MA, USA). In addition, the TG concentration was determined using a commercial kit (Sigma-Aldrich) according to the manufacturer's protocol.

\section{Cholesterol efflux assay}

THP-1 macrophage-derived foam cells were plated in six-well plates and loaded with $1 \mu \mathrm{g} / \mathrm{mL}$ NBD-cholesterol (Invitrogen, Carlsbad, CA, USA) for $4 \mathrm{~h}$. Cells were washed, equilibrated, and maintained with serum-free RPMI 1640 medium containing $0.1 \%$ bovine serum albumin (BSA) for $2 \mathrm{~h}$. Then, cells were incubated with $25 \mu \mathrm{g} / \mathrm{mL}$ apoA-I (Sigma-Aldrich) or $50 \mu \mathrm{g} / \mathrm{mL} \mathrm{HDL}$ (Sigma-Aldrich) at $37^{\circ} \mathrm{C}$ for $4 \mathrm{~h}$. The medium was collected, and the cells were lysed using $0.3 \mathrm{M} \mathrm{NaOH}$ solution. The fluorescence density in the medium and cell lysates was determined using a microplate spectrophotometer. Cholesterol efflux was expressed as percent fluorescence density in medium relative to total fluorescence density (medium + cells).
THP-1 macrophages were incubated with $50 \mu \mathrm{g} / \mathrm{mL}$ acetelyated LDL (ac-LDL, Yiyuan biotechnology) and $5 \mu \mathrm{Ci} / \mathrm{mL}\left[{ }^{3} \mathrm{H}\right]$-cholesterol (PerkinElmer) for $24 \mathrm{~h}$. Cells were washed with PBS and then cultured in RPMI 1640 medium containing $10 \mu \mathrm{M}$ avasimibe (Med Chem Express, NJ, USA) and $0.5 \mathrm{mM}$ 8-bromo-cAMP (8-BrcAMP, Sigma-Aldrich). After $24 \mathrm{~h}$, cells were washed with PBS and maintained in $2 \mathrm{~mL}$ of RPMI 1640 medium containing $1 \mathrm{mg} / \mathrm{mL} \mathrm{BSA}, 25 \mu \mathrm{g} / \mathrm{mL}$ apoA-I, or $50 \mu \mathrm{g} / \mathrm{mL}$ HDL for up to $9 \mathrm{~h}$. The Radioactivity of $\left[{ }^{3} \mathrm{H}\right]$-cholesterol in the medium and cells was measured using a liquid scintillation counter. The percent cholesterol efflux was calculated as the ratio of radioactivity in the medium to total radioactivity (medium + cells).

\section{Dil-ox-LDL uptake}

THP-1 macrophage-derived foam cells were treated with PBS, LV-NC, or LV-CTRP12, followed by incubation with $10 \mu \mathrm{g} / \mathrm{mL}$ Dil-ox-LDL (Yiyuan biotechnology) at $37^{\circ} \mathrm{C}$ for $4 \mathrm{~h}$. After washing with PBS three times, the cells were viewed and imaged by a fluorescence microscope.

\section{Transfection of LXRa small interfering RNA (siRNA) and miR-155-5p mimic/inhibitor}

LXR $\alpha$ siRNA (forward, 5'-GGAUGCUAAUGAAACUGGUTT-3'; reverse, 5'-ACCAGUUUC AUUAGCAUCCGT- $3^{\prime}$ ) and scrambled siRNA as a negative control (forward, 5'-UUCUCCGAA CGUGUCACGUTT-3'; reverse, $5^{\prime}$-ACGUGACACGUUCGGAGAATT-3') were designed and synthesized by GenePharma (Shanghai, China). The siRNAs (100 nM) were transfected into lipidenriched macrophages using Lipofectamine ${ }^{\circledR} 3000$ (Invitrogen) according to the manufacturer's instructions. To determine the impact of gain and loss of function of miR155-5p, THP-1 macrophage-derived foam cells were transfected with $50 \mathrm{nM}$ of miR-155-5p mimic, miR-155-5p inhibitor or their respective negative controls (Ribobio, Guangzhou, China) using Lipofectamine ${ }^{\circledR} 3000$. At $48 \mathrm{~h}$ post-transfection, cells were processed for further analyses.

\section{Bioinformatics prediction and luciferase reporter assay}

The potential targeting sequences between miR-155-5p and LXR $\alpha 3^{\prime}$ untranslated region (UTR) were predicted by miRDB (http://mirdb.org/miRDB/) and TargetScan (http://www.targetscan.org/). The RNAhybrid database (http://bibiserv.techfak.uni-bielefeld.de/rnahybrid/

submission.htmL) was utilized to calculate the free energy score. Dual luciferase reporter assay was performed using 293T cells. Cells were seeded in a 96-well plate at a density of 15,000 cells per well and maintained in serumfree media for $2 \mathrm{~h}$ before transfection. Then, cells were cotransfected with miR-155-5p mimic or mimic control and reporter plasmid pmirGLO (Promega) containing predicted binding sequences or mutant sequences of LXR $\alpha$ 
with miR-155-5p using Lipofectamine ${ }^{\circledR}$ 3000. After $48 \mathrm{~h}$ of transfection, cells were harvested, and the luciferase activity was detected using a Luciferase detection assay kit (KeyGen Biotech, Nanjing, China).

\section{Assessment of atherosclerotic lesions in the aortic root}

Mice were euthanized, and the upper part of the heart and proximal aorta were dissected carefully. After rinsing with PBS, samples were embedded in Optimal Cutting Temperature compound (OCT, Sakura Finetek Japan Co., Ltd, Tokyo, Japan) and stored at $-80^{\circ} \mathrm{C}$. Serial $8-\mu \mathrm{m}$-thick cryosections throughout the three aortic valves were obtained and placed on glass slides using a cryostat microtome (Leica CM3050 S). Sections were then stained with Oil Red O, hematoxylin-eosin (HE), and Masson. Quantitative analyses were performed using Image-Pro Plus 7.0 software.

\section{Detection of plasma lipid levels}

Blood samples were collected from the retro-orbital plexus of $\mathrm{apoE}^{-1-}$ mice. Plasma levels of TC, HDLcholesterol (HDL-C), low-density lipoprotein cholesterol (LDL-C), and TG were determined using the commercial kits (Sigma-Aldrich).

\section{In vivo reverse cholesterol transport (RCT) assay}

The in vivo RCT assay was performed as previously described $^{27}$. J774 macrophages were treated with $50 \mu \mathrm{g} /$ $\mathrm{mL}$ ac-LDL and $5 \mu \mathrm{Ci} / \mathrm{mL}\left[{ }^{3} \mathrm{H}\right]$-cholesterol for $48 \mathrm{~h}$. The labeled cells were resuspended in ice-cold Dulbecco's modified Eagle's medium (DMEM, ThermoFisher, Scotts Valley, CA, USA) and intraperitoneally injected into apoE $^{-1-}$ mice $\left(5 \times 10^{6}\right.$ cells/mouse, $n=5$ per group $)$. At 6 , 24 , and $48 \mathrm{~h}$ after injection, plasma samples were collected and the radioactivity in $10 \mu \mathrm{L}$ aliquots was evaluated by a liquid scintillation counter. The total feces were collected continuously until $48 \mathrm{~h}$, vacuum dried and homogenized in $50 \% \mathrm{NaOH}$ overnight. Then, $20 \mu \mathrm{L}$ aliquots were used for scintillation counting. At the end of the study, mice were sacrificed, and the liver samples were harvested. The hepatic tissue $(100 \mathrm{mg})$ was mixed with hexane/isopropanol (3:2) for $48 \mathrm{~h}$ and then dried overnight. Lipids were resolubilized in liquid scintillation fluid and radioactivity was counted. The RCT efficiency was estimated as the ratio of radioactivity in the plasma, liver, or feces to total radioactivity injected at baseline.

\section{RNA isolation and qRT-PCR}

Total RNA was obtained from cultured cells and the tissues using TRIzol reagent according to the manufacturer's instructions (ThermoFisher). The purity of the extracted RNA was assessed using a Nanodrop 3000 (ThermoFisher). The first strand of complementary DNA was synthesized using a high-capacity cDNA reverse transcription kit (Takara, Kyoto, Japan). Then, qRT-PCR was conducted utilizing $\mathrm{SYBR}^{\circledR}$ Premix Ex TaqTM II reagent kit (Takara) on an ABI 7900HT Fast Real-Time PCR System (Applied Biosystems, Foster City, CA, USA) for 40 cycles $\left(95^{\circ} \mathrm{C}\right.$ for $3 \mathrm{~min}, 90^{\circ} \mathrm{C}$ for $15 \mathrm{~s}$, and $60^{\circ} \mathrm{C}$ for $1 \mathrm{~min})$. The primer sequences for qRT-PCR are listed in Supplementary Table 1, which were designed and synthesized by Sangon (Shanghai, China). U6 was selected as internal control for miR-155-5p and $\beta$-actin for all others. The specificity of all PCR products was checked using melting curve analysis. Relative gene expression was determined by the $2^{-\Delta \Delta \mathrm{Ct}}$ method.

\section{Western blot analysis}

The cultured cells and tissues were lysed by RIPA buffer (Beyotime) mixed with $0.1 \mathrm{mmol} / \mathrm{L}$ phenylmethylsulfonyl fluoride. The concentration of total proteins in the extracts was quantified using a BCA protein assay kit. Then, proteins were subjected to SDS-PAGE, followed by immunoblotting with rabbit polyclonal antibody against CTRP12 (PA5-46452, 1:500, ThermoFisher), rabbit polyclonal antibody against ABCA1 (PA1-16789, 1:800, ThermoFisher), rabbit polyclonal antibody against ABCG1 (GTX30598, 1:500, GeneTex, Irvine, CA, USA), rabbit polyclonal antibody against CD36 (PA1-16813, 1:1000, ThermoFisher), mouse monoclonal antibody against SR-A (sc-166184, 1:500, Santa Cruz, TX, USA), rabbit polyclonal antibody against LXR $\alpha$ (L5044, 1:1000, Sigma-Aldrich) and rabbit monoclonal antibody against $\beta$-actin (ab115777, 1:1000, Abcam, Cambridge, MA, USA). After a series of rinses with PBS-T, the membranes were further incubated with HRP-labeled secondary antibodies (1:5000, Beyotime). The protein bands were visualized using Tanon 5500 (Shanghai, China) and BeyoECL Plus (Beyotime). The densitometry values were determined using Image $J$ software and normalized to $\beta$-actin values.

\section{Enzyme-linked immunosorbent assay (ELISA)}

Serum monocyte chemotactic protein-1 (MCP-1), tumor necrosis factor- $\alpha$ (TNF- $\alpha$ ), and interleukin-10 (IL-10) levels were measured by the commercial ELISA kits (R\&D systems, Minneapolis, MN, USA) according to the manufacturer's protocol. In brief, $100 \mu \mathrm{L}$ serum sample or standard preparation were added into the wells and incubated at $37^{\circ} \mathrm{C}$ for $120 \mathrm{~min}$. Thereafter, $100 \mu \mathrm{L}$ biotinconjugated antibody was added into each well. After $90 \mathrm{~min}$ of incubation, the substrate solution was added into each well and sustained for $30 \mathrm{~min}$. After washing five times with TBS, TMB was added and sustained for $30 \mathrm{~min}$ in the dark. The absorbance at $450 \mathrm{~nm}$ was detected using the iMark ${ }^{\mathrm{TM}}$ Microplate Reader (Bio-Rad, Hercules, CA, USA).

\section{Statistical analysis}

All data are represented as the mean \pm standard deviation (SD) from three independent experiments. 
Differences between two groups were compared by an unpaired Student's $t$ test. One-way ANOVA was used to compare the differences among multiple groups. Statistical analyses were conducted using GraphPad Prism 9.0 software. A $P$ value less than 0.05 was considered statistically significant.

\section{Results}

CTRP12 inhibits lipid accumulation and promotes cholesterol efflux from macrophages

Macrophage lipid accumulation is a critical event during atherogenesis. To explore the effects of CTRP12 on lipid accumulation, THP-1 macrophage-derived foam cells were treated with PBS, LV-NC, or LV-CTRP12. As shown in Fig. 1A, transfection with LV-CTRP12 elevated the protein levels of CTRP12 by $\sim 3.5$ folds compared with control group, while LV-NC did not alter its expression. In comparison with unloaded cells, THP-1 macrophagederived foam cells displayed a significant increase in intracellular TC, FC, CE, and TG contents, and this increase was attenuated by LV-CTRP12 treatment (Fig. $1 \mathrm{~B}, \mathrm{C})$. Accordingly, the Oil Red $\mathrm{O}$ staining results showed that CTRP12 overexpression reduced intracellular lipid droplets (Fig. 1D). It is well known that lipid accumulation is caused by decreased cholesterol efflux and/or increased cholesterol uptake ${ }^{28}$. Next, we investigated whether CTRP12 could affect these two processes. As expected, CTRP12 overexpression facilitated the export of NBD-cholesterol from THP-1 macrophagederived foam cells to apoA-I (Fig. 1E) and HDL (Fig. 1F). Both avasimibe, a potent inhibitor of acyl-coenzyme A: cholesterol acyltransferase (ACAT), and 8-Br-cAMP, a cAMP activator, have been shown to enhance cholesterol efflux capacity ${ }^{29,30}$. In THP-1 macrophages loaded with ac-LDL, LV-CTRP12 treatment also increased the efficiency of $\left[{ }^{3} \mathrm{H}\right]$-cholesterol efflux to apoA-I and HDL in the presence of avasimibe and 8-Br-cAMP (Supplementary Fig. 1). However, there was no significant difference in the uptake of Dil-ox-LDL by THP-1 macrophagederived foam cells between control group and LVCTRP12 group (Fig. 1G). Collectively, these results suggest that CTRP12 ameliorates lipid accumulation by promoting cholesterol efflux from macrophages.

\section{CTRP12 upregulates $A B C A 1$ and $A B C G 1$ expression in macrophages}

Intracellular cholesterol efflux is predominantly mediated by ABCA1 and ABCG1. Both CD36 and SR-A are associated with cholesterol uptake ${ }^{31}$. To clarify the underlying mechanisms by which CTRP12 regulates lipid accumulation, both qRT-PCR and western blot were used to measure the expression of these cholesterol efflux and uptake markers. As expected, incubation with LVCTRP12 markedly elevated the mRNA and protein levels of ABCA1 and ABCG1 in THP-1 macrophagederived foam cells (Fig. 2A, B). However, CTRP12 overexpression had no impact on CD36 and SR-A expression (Fig. 2C, D). These findings suggest that CTRP12 promotes cholesterol efflux by upregulating $\mathrm{ABCA} 1$ and ABCG1 expression.

\section{LXRa is involved in CTRP12-induced upregulation of $A B C A 1$ and $A B C G 1$ expression}

LXR $\alpha$ is the most important transcription factor to stimulate $A B C A 1$ and $A B C G 1$ expression. We wondered whether LXR $\alpha$ plays a role in the regulation of ABCA1 and ABCG1 expression by CTRP12. To this end, we first treated THP-1 macrophage-derived foam cells with LVCTRP12 and found that CTRP12 overexpression increased the mRNA and protein levels of LXR $\alpha$ (Fig. 3A). Subsequently, THP-1 macrophage-derived foam cells were transfected with scrambled siRNA or LXR $\alpha$ siRNA. When compared with the control group, transfection with LXR $\alpha$ siRNA led to a marked decrease in LXR $\alpha$ protein expression (Fig. 3B). Finally, THP-1 macrophage-derived foam cells were transfected with LXR $\alpha$ siRNA, which was followed by incubation with or without LV-CTRP12. Knockdown of LXR $\alpha$ with siRNA reduced the effects of CTRP12 on ABCA1 and ABCG1 expression (Fig. 3C). Consistently, CTRP12-induced enhancement of NBDcholesterol efflux to apoA-I and HDL was reversed by LXR $\alpha$ siRNA (Fig. 3D, E). All these findings support the notion that CTRP12 increases ABCA1 and ABCG1 expression in an LXR $\alpha$-dependent manner.

\section{Identification of LXRa as a direct target of miR-155-5p}

Bioinformatics analyses (Targetscan and miRDB) for miRNA recognition sequences on LXR $\alpha$ revealed the presence of a putative miR-155-5p binding site (Fig. 4A). Further, the RNAhybrid database showed a lower free energy score $(-19.0 \mathrm{kcal} / \mathrm{mol})$, suggesting that $\mathrm{miR}-155$ $5 p$ can bind stably to LXR $\alpha 3^{\prime}$ UTR (Fig. 4B). To further confirm the target prediction algorithms, we constructed a luciferase reporter plasmid containing either wild-type miR-155-5p binding site (LXR $\alpha$-WT) or corresponding mutant (LXR $\alpha$-Mut) (Fig. 4A). These plasmids were transfected into 293T cells together with miR-155-5p mimic or mimic control, followed by luciferase reporter assay. Co-transfection of LXR $\alpha-W T$ and miR-155-5p mimic markedly diminished the luciferase activity, while this effect disappeared when the miR-155-5p binding site was mutated (Fig. 4C). We then transfected THP-1 macrophage-derived foam cells with mimic control, miR-155-5p mimic, inhibitor control, and miR-155-5p inhibitor, respectively. In comparison with the control group, miR-155-5p mimic treatment elevated miR-155$5 p$ levels by 7.1-fold, and miR-155-5p inhibitor suppressed miR-155-5p expression by 68\% (Fig. 4D), 
A

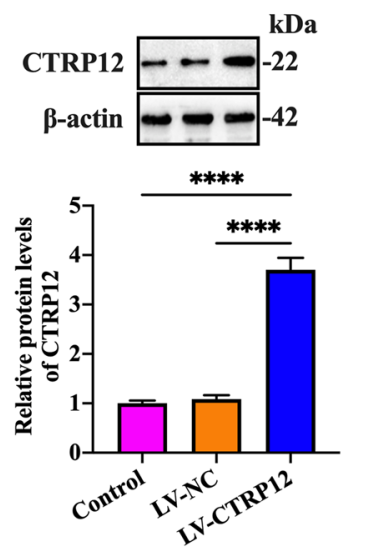

B

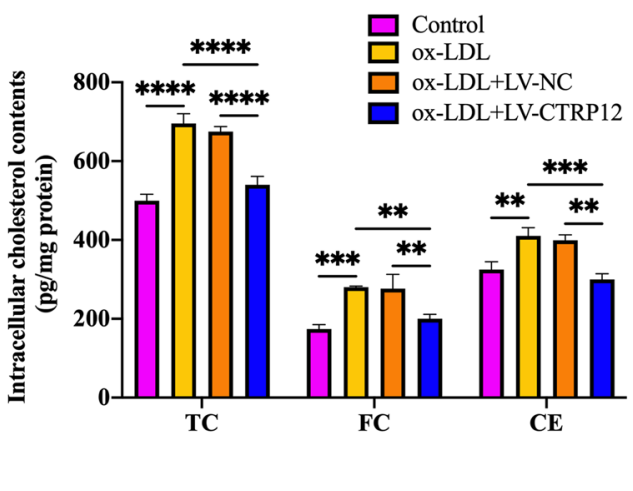

C

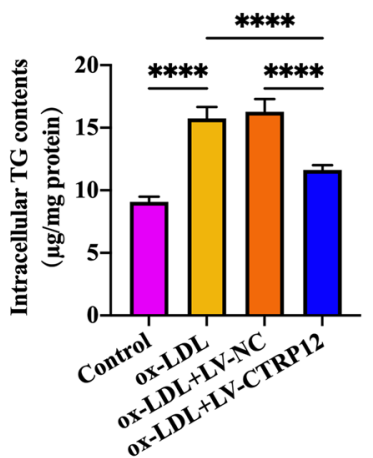

D
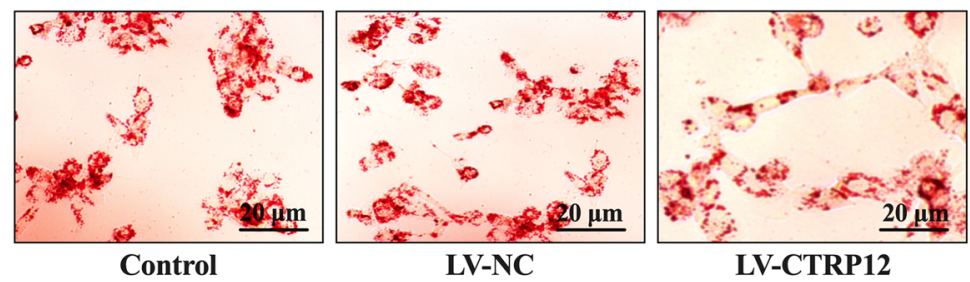

E

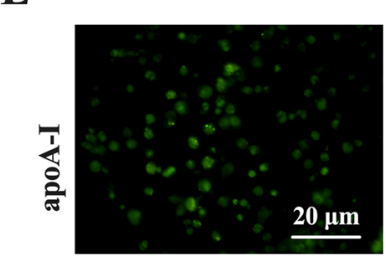

F

Control

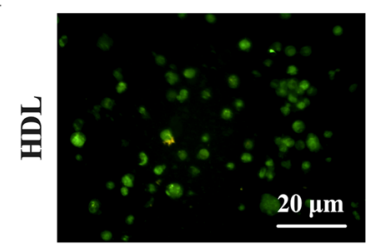

Control

G

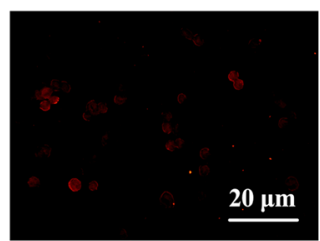

Control

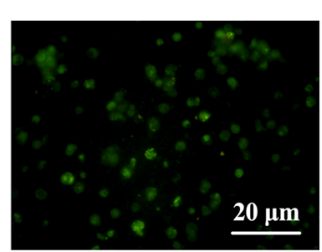

LV-NC

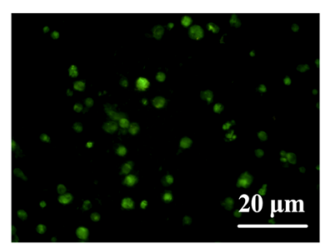

LV-NC

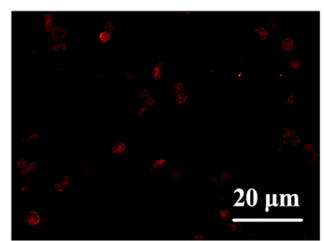

LV-NC

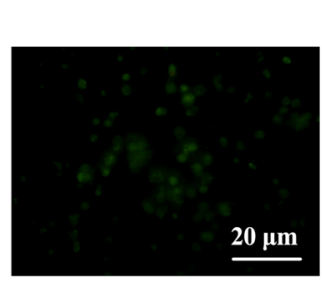

LV-CTRP12

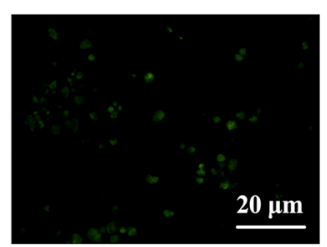

LV-CTRP12

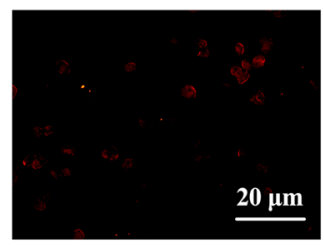

LV-CTRP12

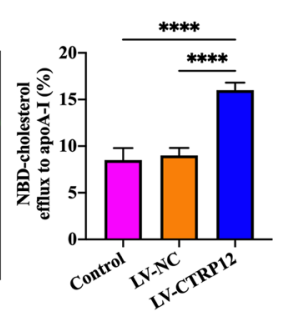

Fig. 1 Effects of CTRP12 on cholesterol efflux and lipid accumulation in macrophages. A-G THP-1 macrophages were pretreated with or without $50 \mu \mathrm{g} / \mathrm{mL}$ ox-LDL for $48 \mathrm{~h}$, and then transduced with PBS, LV-NC, or LV-CTRP12 for $72 \mathrm{~h}$. A CTRP12 expression was determined by western blot. B, C Measurement of intracellular TC, FC, CE, and TG concentrations. D Representative images of Oil red O staining ( $\times 200)$. Scale bar $=20 \mu$ m. $\mathbf{E}$ $\mathbf{F}$ Representative fluorescent images of NBD-cholesterol burden $(\times 200)$. Cholesterol efflux mediated by apoA-I and HDL was quantified in these groups. Scale bar $=20 \mu \mathrm{m}$. G Representative fluorescent images of Dil-ox-LDL uptake $(\times 200)$. Scale bar $=20 \mu \mathrm{m}$. Data are expressed as the mean \pm SD from three independent experiments. ${ }^{* *} P<0.01$, ${ }^{* * *} P<0.001,{ }^{* * *} P<0.0001$. 


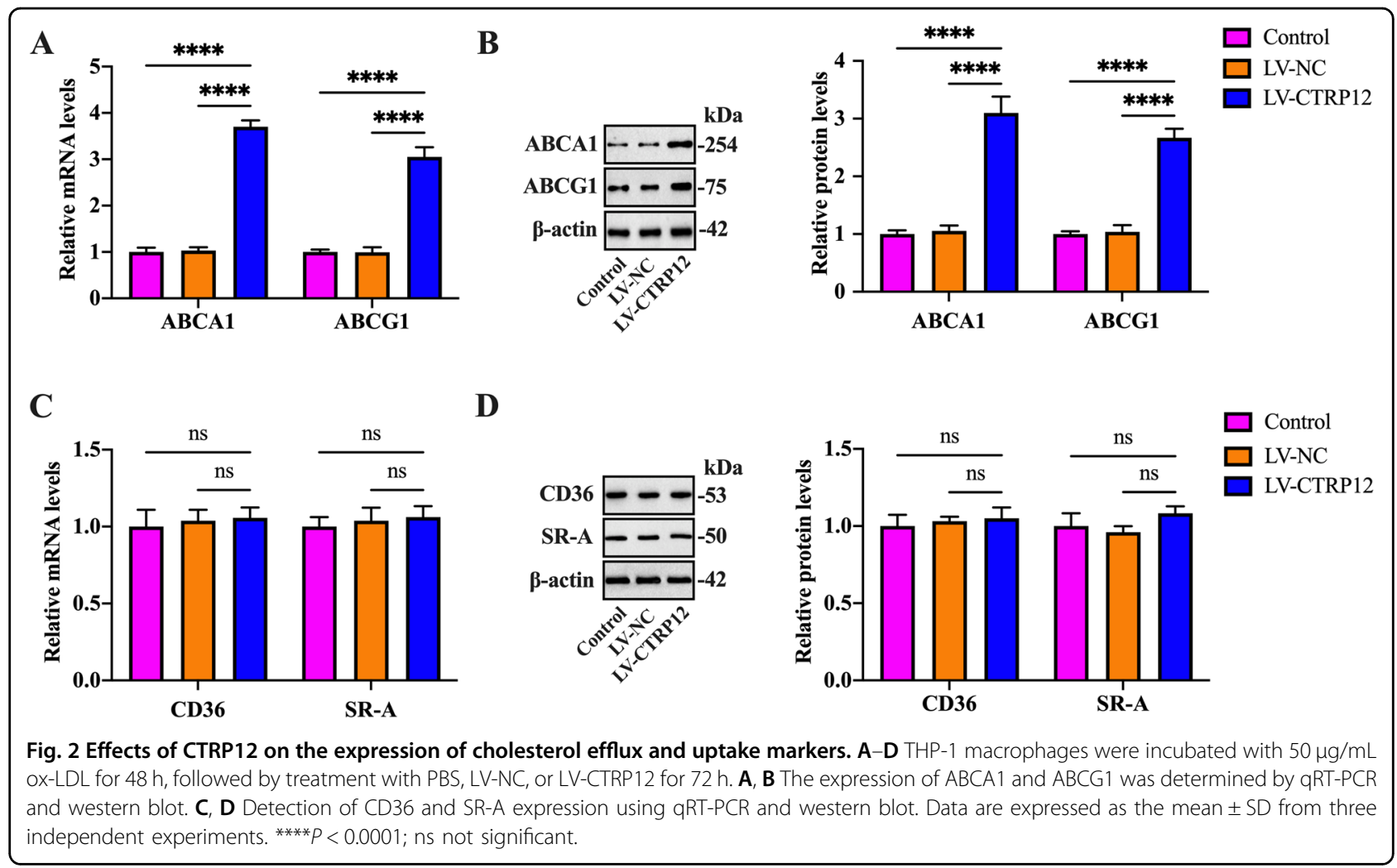

showing a high transfection efficacy. Meanwhile, transfection with miR-155-5p mimic decreased the mRNA and protein levels of $L X R \alpha$, while miR-155-5p inhibitor had an opposite effect (Fig. 4E). Overall, these data support the concept that miR-155-5p can directly interact with LXR $\alpha 3^{\prime}$ UTR and negatively modulate its expression at the post-transcriptional level.

\section{MiR-155-5p is required for CTRP12-induced upregulation of $L X R a, A B C A 1$, and ABCG1 expression}

The above studies have identified LXR $\alpha$ as a direct target of miR-155-5p. We speculated that CTRP12induced upregulation of $\mathrm{LXR} \alpha, \mathrm{ABCA} 1$, and $\mathrm{ABCG} 1$ expression is likely mediated by miR-155-5p. To test this possibility, we first detected miR-155-5p expression using qRT-PCR in THP-1 macrophage-derived foam cells treated with LV-CTRP12. Our results indicated that CTRP12 overexpression markedly attenuated miR155-5p levels (Fig. 5A). Subsequently, THP-1 macrophage-derived foam cells were transfected with miR155-5p mimic prior to treatment with LV-CTRP12. As shown in Fig. 5B-D, treatment with LV-CTRP12 alone upregulated $\mathrm{LXR} \alpha, \mathrm{ABCA} 1$, and $\mathrm{ABCG} 1$ expression, and these effects were reversed by miR-155-5p mimic. These data suggest the involvement of miR-155-5p in CTRP12-induced enhancement of LXR $\alpha, A B C A 1$, and ABCG1 expression.
CTRP12 attenuates inflammatory response through the miR-155-5p/LXRa pathway in macrophages

The atheroprotective role of LXR $\alpha$ is not only due to its influence on cholesterol efflux, but also on inflammatory response. Given CTRP12 as an activator of $\mathrm{LXR} \alpha$, we examined the impact of CTRP12 on macrophage polarization and inflammatory cytokine expression using qRT-PCR. As shown in Fig. 6A, treatment with LV-CTRP12 downregulated the mRNA expression of M1 markers inducible nitric oxide synthase (iNOS) and CD86 but upregulated the mRNA expression of M2 markers of mannose receptor $\mathrm{C}$ type 1 (Mrc-1) and arginase-1 (Arg-1) in THP-1 macrophage-derived foam cells. Consistently, CTRP12 overexpression decreased the mRNA levels of pro-inflammatory cytokines MCP-1 and TNF- $\alpha$ but increased the mRNA levels of anti-inflammatory cytokine IL-10 (Fig. 6B). The effects of CTRP12 on the mRNA expression of iNOS, CD86, Mrc-1, Arg-1, MCP-1, TNF- $\alpha$, and IL-10 was significantly reversed by LXR $\alpha$ siRNA (Fig. 6C, D) and miR-155-5p mimic (Fig. 6E, F). To further confirm the data obtained using THP-1 macrophage-derived foam cells, we isolated MPMs from apoE ${ }^{-1-}$ mice and found that CTRP12 overexpression attenuated the mRNA levels of iNOS, CD86, MCP-1, and TNF- $\alpha$ but elevated the mRNA levels of Mrc-1, Arg-1, and IL-10 (Supplementary Fig. 2A, B). Pretreatment with LXR $\alpha$ siRNA or miR-155-5p mimic also diminished the influence of CTRP12 on the mRNA expression of iNOS, 


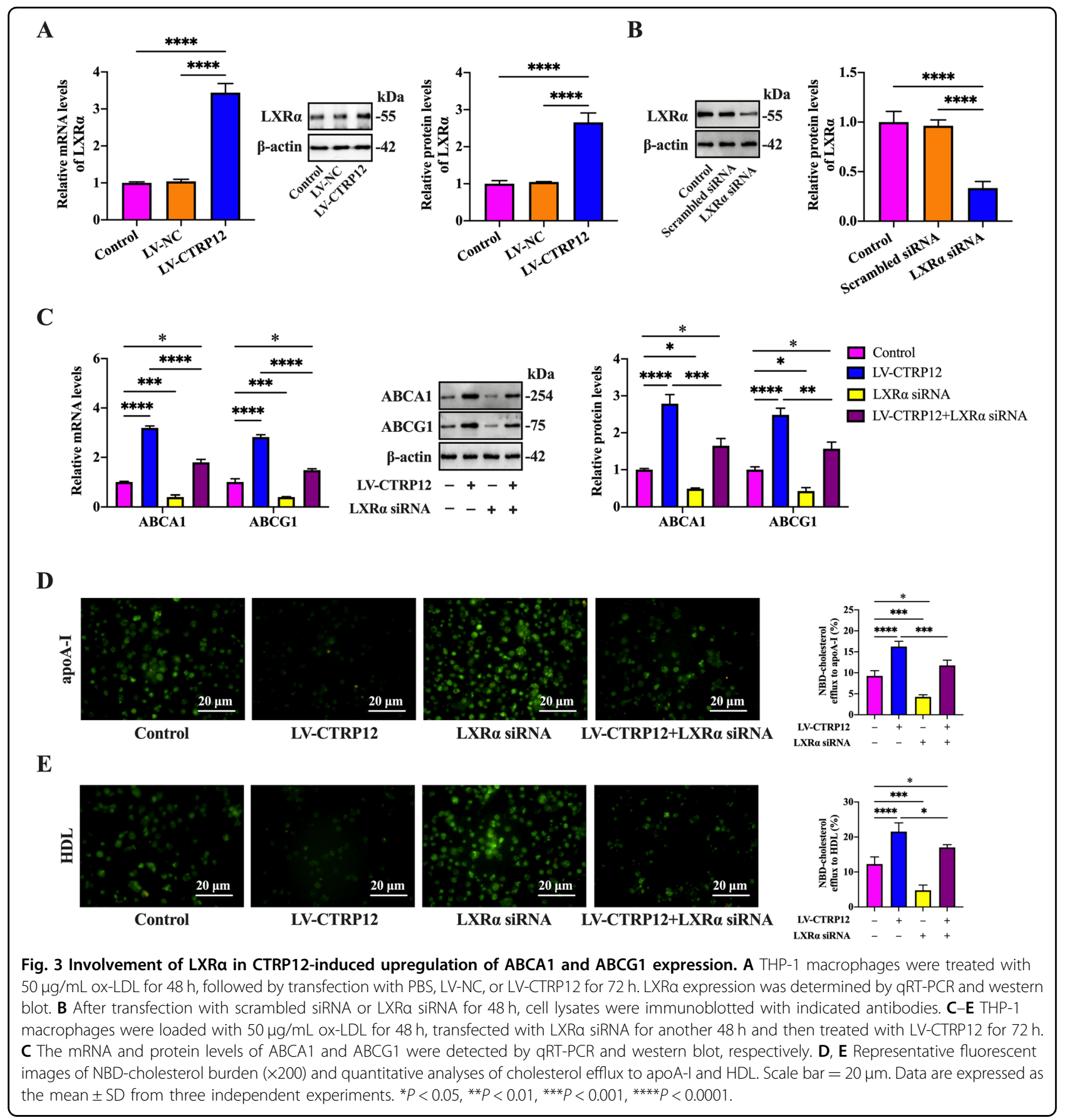

CD86, Mrc-1, Arg-1, MCP-1, TNF- $\alpha$, and IL-10 (Supplementary Fig. 2C-F). Collectively, these observations suggest that CTRP12 exerts an anti-inflammatory effect by promoting M2 macrophage polarization via the miR-155-5p/ LXR $\alpha$ signaling pathway.

\section{CTRP12 inhibits the development of atherosclerosis in apoE $^{-1-}$ mice}

Finally, to determine the role of CTRP12 in atherosclerosis in vivo, apoE $\mathrm{E}^{-1-}$ mice were fed a Western diet for 12 weeks and simultaneously injected with PBS, LVNC or LV-CTRP12. At the end of the study, about 4.1fold elevation of CTRP12 protein levels was observed in the aortas from apoE $\mathrm{E}^{-/-}$mice transduced with LVCTRP12 compared with control mice (Fig. 7A). Meanwhile, the number and size of atherosclerotic lesions in the aortic arch regions was significantly decreased in LVCTRP12-treated group compared with control group (Fig. 7B). HE, Oil Red O and Masson staining of cross-sections of the aortic root showed that CTRP12 overexpression 


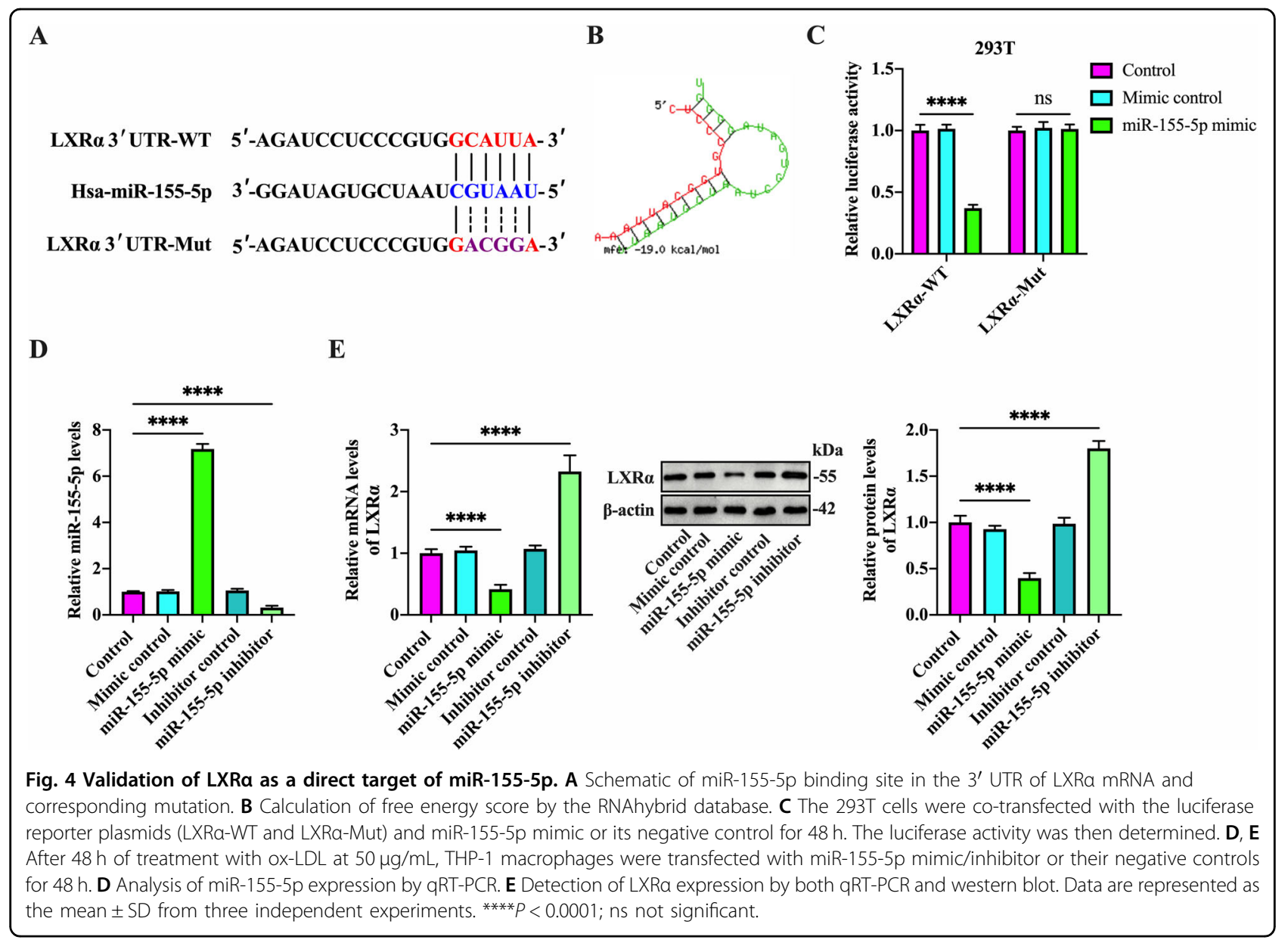

markedly decreased lesion area and inhibited lipid deposition, with no impact on collagen content (Fig. 7C). These in vivo results indicate that CTRP12 plays a protective role in the development of atherosclerosis.

To elucidate the underlying mechanisms by which CTRP12 protects against atherosclerosis in vivo, we first detected the cholesterol and TG concentrations in MPMs. Our results revealed that ox-LDL loading enhanced the amounts of TC, FC, CE, and TG, which was prevented by LV-CTRP12 transduction (Supplementary Fig. 3A, B). Subsequently, we examined the effects of CTRP12 on plasma lipid profile and RCT. Figure 8A shows that injection of LV-CTRP12 elevated HDL-C levels but had no impact on TC, LDL-C, and TG levels. Consistently, apoE ${ }^{-/-}$mice injected with LV-CTRP12 exhibited a significant increase of $\left[{ }^{3} \mathrm{H}\right]-$ cholesterol content in the plasma, liver, and feces (Fig. $8 \mathrm{~B})$, suggesting a promotive effect of CTRP12 on RCT. In line with the findings of in vitro experiments, LVCTRP12 injection decreased miR-155-5p levels but enhanced the expression of LXR $\alpha, A B C A 1$, and ABCG1 in the aortas of apoE ${ }^{-1-}$ mice (Fig. 8C, D). Meanwhile, CTRP12 overexpression downregulated iNOS, CD86,
MCP-1, and TNF- $\alpha$ expression but upregulated Mrc-1, Arg-1, and IL-10 expression (Supplementary Fig. 4A, B). CTRP12 overexpression also led to a significant decrease in serum MCP-1 and TNF- $\alpha$ levels and an increase in serum IL-10 levels (Supplementary Fig. 4C). Taken together, all these data demonstrate that the antiatherogenic action of CTRP12 is attributed to its abilities to promote RCT and suppress vascular inflammation.

\section{Discussion}

As a paralog of adiponectin, CTRP12 is composed of a signal peptide, a short $\mathrm{N}$-terminal domain, a collagen-like domain and a globular C1q-like domain. Adiponectin is known to protect against atherosclerosis in animal mod$\mathrm{els}^{32,33}$. Although CTRP12 was reported to participate in the occurrence and development of cardiovascular disease $^{22-24}$, its role in atherogenesis is still largely unknown. Our in vivo experiments demonstrated that aortic plaque burden was significantly lower in apoE ${ }^{-1-}$ mice overexpressing CTRP12 than that in apoE ${ }^{-1-}$ littermates. This is the first study to report an association between CTRP12 and atherosclerosis. 


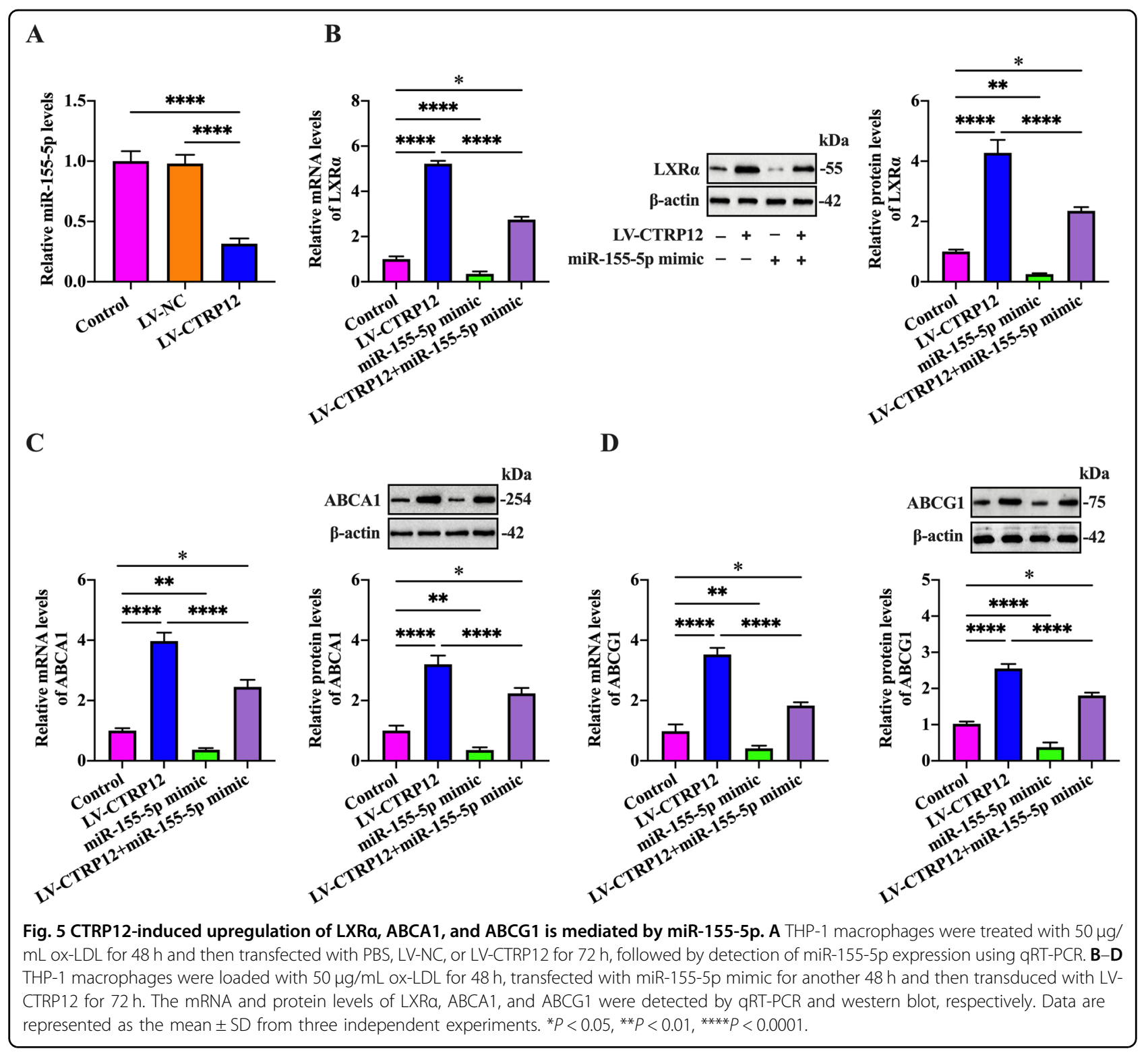

Lipid metabolism disorder plays a critical role in the pathogenesis of atherosclerosis. An epidemiological survey showed that a $1 \mathrm{mg} / \mathrm{dL}$ elevation of plasma HDL-C levels decreases the risk of coronary heart disease in men by $2 \%$ and women by $3 \%^{34}$. RCT, a process in which excessive peripheral cholesterol is transported by HDL to the liver for excretion into the bile and feces, is proposed to be a major mechanism for the antiatherogenic action of HDL. In our previous studies, administration of mangiferin, a xanthonoid extracted from Salacia oblonga, is protective against atherosclerosis by elevating plasma HDL-C levels and promoting RCT in apoE ${ }^{-1-}$ mice $^{35}$. CTRP12 increases insulin sensitivity and improves glucose metabolism ${ }^{36,37}$. Partial deficiency of CTRP12 in male mice fed a high-fat diet impairs lipid clearance from the body and consequently augments hepatic cholesterol content $^{38}$. In patients with coronary artery disease, circulating CTRP12 concentration is positively correlated with HDL-C levels ${ }^{24}$. Here, we found that overexpression of CTRP12 led to a significant increase in circulating HDL-C levels and RCT efficiency, which was accompanied by decreased lipid deposition within the plaques. Thus, CTRP12 exerts a beneficial impact on lipid metabolism, which contributes to its atheroprotective effect.

Excessive lipid deposition in macrophages results in foam cell formation, which is regarded as a critical event during atherogenesis. There is increasing evidence that adiponectin inhibits lipid accumulation and subsequent transformation of macrophages into foam cells ${ }^{39-41}$. Similarly, treatment with recombinant CTRP12 suppresses 


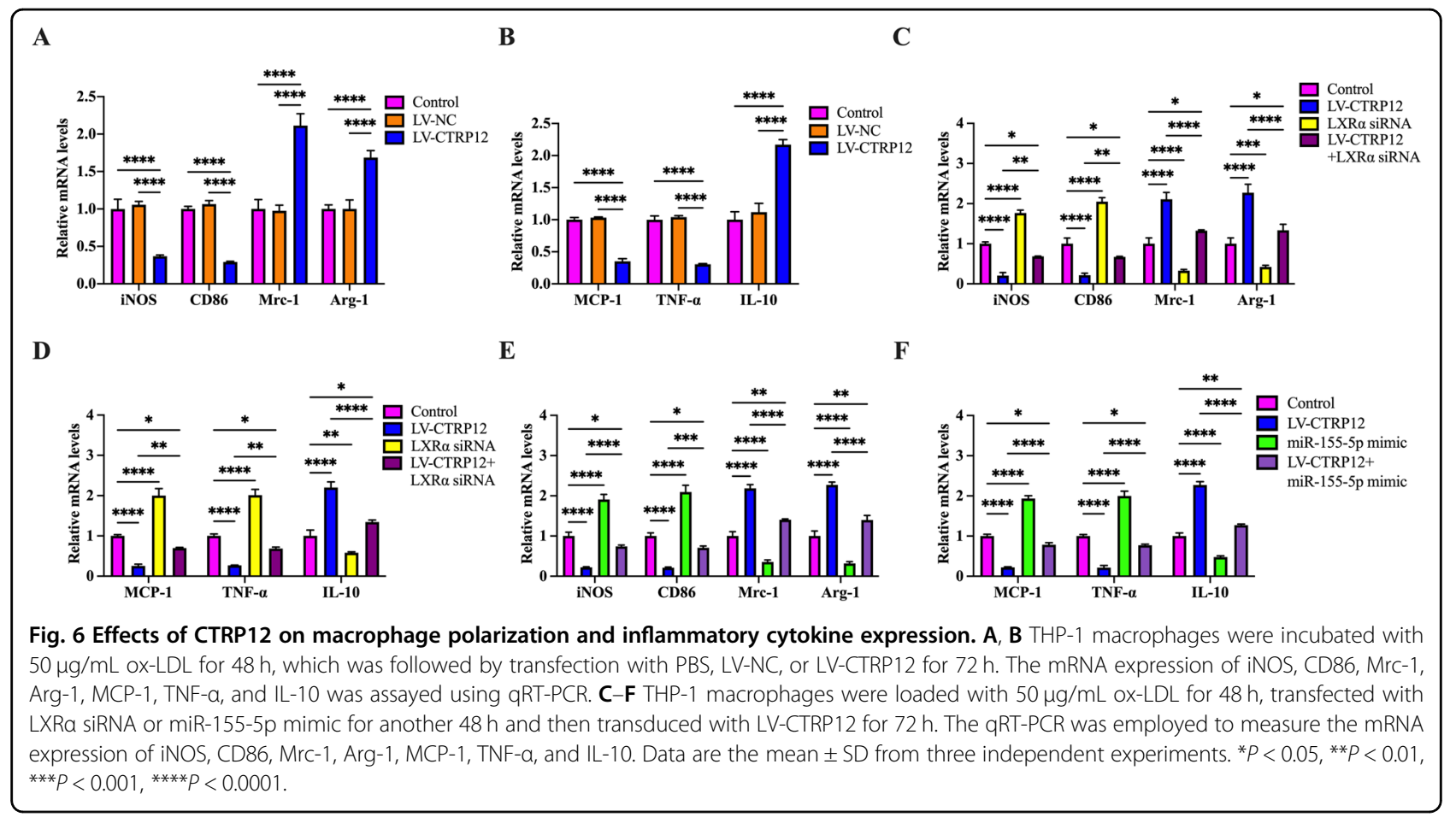

lipogenesis in rat H4IIE hepatoma cells ${ }^{42}$. In this study, we found that overexpression of CTRP12 significantly reduced intracellular lipid droplets and decreased cholesterol and TG contents in macrophages. These data suggest that CTRP12 contributes to alleviation of macrophage lipid accumulation.

$A B C A 1$ and $A B C G 1$ belong to the members of the $A B C$ superfamily and promote cholesterol efflux to apoA-I and HDL, respectively. It is estimated that they are responsible for $\sim 70 \%$ of cholesterol release from lipid-loaded macrophages $^{43}$. Our groups and others have revealed that administration of fargesin, mangiferin, or polydatin inhibits macrophage lipid accumulation and atherosclerotic plaque formation by facilitating ABCA1- and ABCG1dependent cholesterol efflux ${ }^{6,35,44}$. SR-A and CD36 are the members of the scavenger receptor family and account for $75-90 \%$ of ox-LDL internalization by macrophages $^{45}$. Extensive studies have demonstrated that prevention of SR-A and/or CD36 expression contributes to alleviation of lipid deposition in macrophages ${ }^{46-48}$. In this study, we observed that treatment with LV-CTRP12 markedly upregulated ABCA1 and ABCG1 expression in THP-1 macrophage-derived foam cells, which was accompanied by a significant increase in cholesterol efflux toward apoA-I and HDL. Thus, promotion of ABCA1and ABCG1-mediated cholesterol efflux is a key mechanism by which CTRP12 suppresses macrophage lipid accumulation and mitigates atherosclerosis. Notably, Dil-ox-LDL internalization and the expression of SR-A and CD36 was not affected by CTRP12 overexpression, suggesting that CTRP12-induced alleviation of lipid accumulation is not due to decreased cholesterol uptake.

LXR $\alpha$ is a nuclear hormone receptor that is activated by oxysterols and endogenous oxidative metabolites of cholesterol. After forming an obligate heterodimer with retinoid X receptor, LXR $\alpha$ binds to LXR-response elements in the promoter of $A B C A 1$ and $A B C G 1$ genes to stimulate their transcription ${ }^{49}$. Studies from our group and others have revealed that angiopoietin-1, pregnancyassociated plasma protein- $\mathrm{A}$, and homocysteine inhibit ABCA1- and ABCG1-dependent cholesterol efflux from lipid-loaded macrophages and aggravate atherosclerosis in $\mathrm{apoE}^{-1-}$ mice by downregulating $\mathrm{LXR} \alpha$ expression ${ }^{50-52}$. As a pleiotropic miRNA, miR-155-5p is linked to metabolic diseases, such as diet-induced obesity ${ }^{53}$ and diabetes $^{54}$. A recent study showed that metastasis-associated lung adenocarcinoma transcript 1 , a long noncoding RNA (lncRNA), enhances nuclear factor I/A expression by competitively binding to miR-155-5p, thereby blocking ox-LDL-stimulated dendritic cell maturation and attenuating atherosclerosis in $\mathrm{apoE}^{-1-}$ mice $^{55}$. This finding reveals a proatherogenic effect of miR-155-5p. In the current studies, we identified LXR $\alpha$ as a direct target of miR-155-5p, as evidenced by bioinformatics prediction and luciferase reporter assay. Transduction with LVCTRP12 was shown to upregulate LXR $\alpha$ expression and attenuate miR-155-5p levels in THP-1 macrophagederived foam cells and the aortas from apoE ${ }^{-1-}$ mice. Further, pretreatment with LXR $\alpha$ siRNA or miR-155-5p mimic reduced the effects of LV-CTRP12 on ABCA1 and 
A

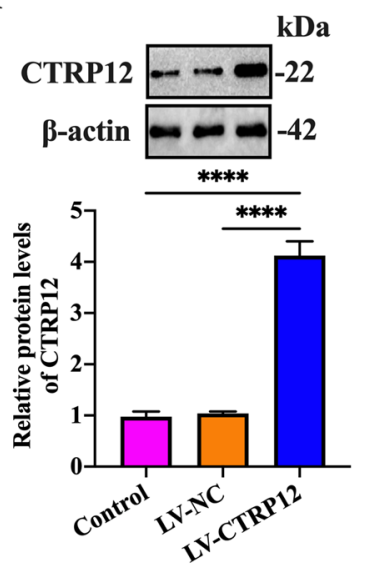

C
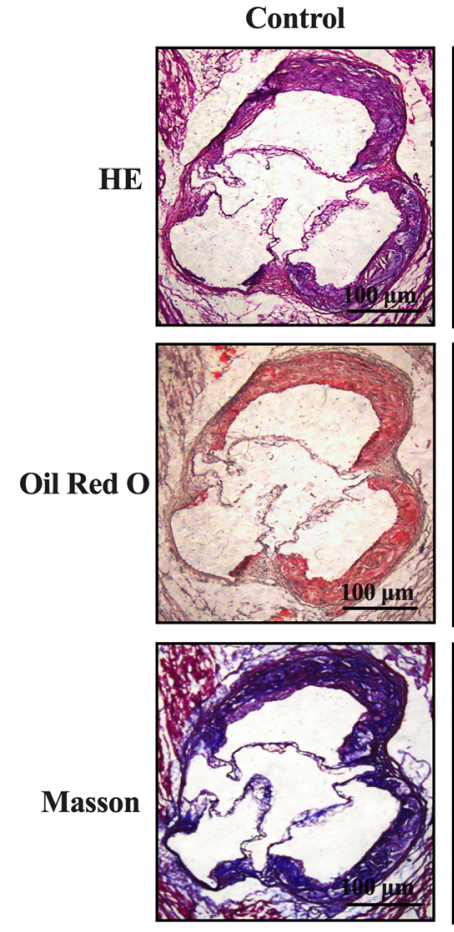

B

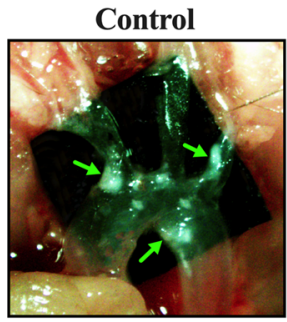

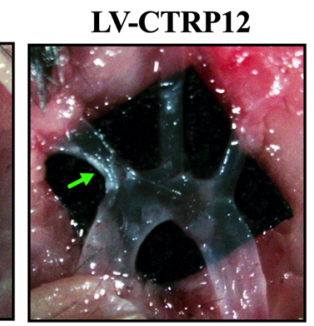

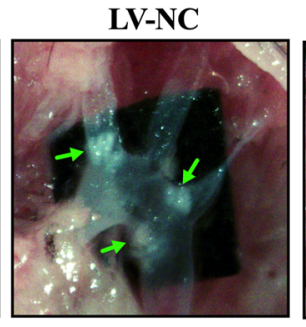

LV-NC
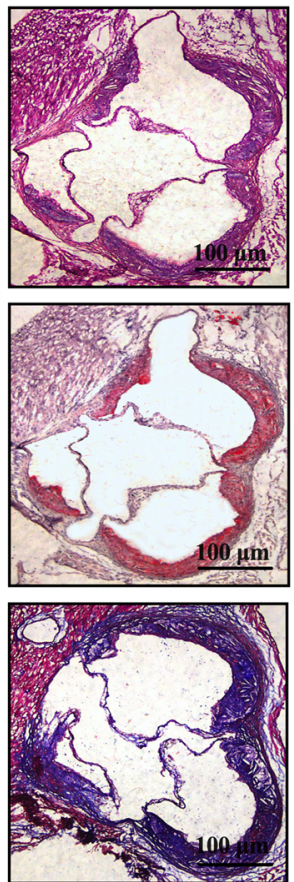

LV-CTRP12
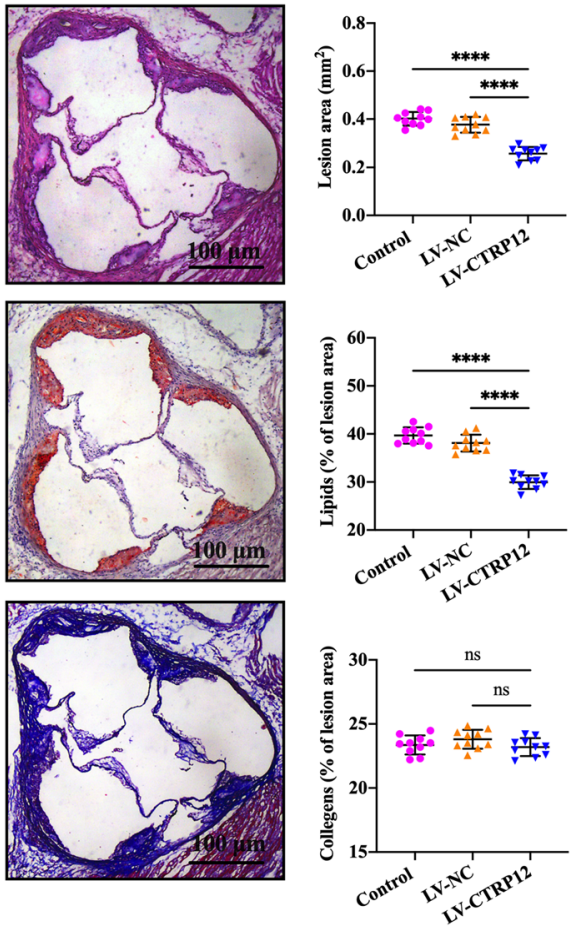

Fig. 7 CTRP12 inhibits atherosclerosis in apoE ${ }^{-1-}$ mice. A-C Western diet-fed apoE ${ }^{-1-}$ mice were injected via the tail vein with PBS, LV-NC, or LVCTRP12 ( $n=15$ in each group). A Analysis of CTRP12 expression in the aortas by western blot $(n=10)$. B The plaques (green arrows) in the aortic arch under a stereoscopic microscope $(n=10)$; C Cryosections of the aortic root were stained with HE, Oil Red O, or Masson, followed by quantification of lesion area, lipid accumulation and collagen contents using Image-Pro Plus 7.0 software $(n=10)$. Scale bar $=100 \mu \mathrm{m}$. Data are represented as the mean \pm SD. ${ }^{* * *} P<0.0001$; ns not significant.

ABCG1 expression, suggesting that CTRP12-induced enhancement of ABCA1 and ABCG1 expression and promotion of cholesterol efflux is mediated by the miR$155-5 \mathrm{p} / \mathrm{LXR} \alpha$ pathway.

Atherosclerosis is regarded as a lipid-driven inflammatory disease. Under inflammatory conditions, macrophages can polarize into two major phenotypes called classically activated M1 macrophages and alternatively activated M2 macrophages. M1 macrophages secrete proinflammatory cytokines and promote the development of atherosclerosis, while M2 macrophages contribute to inflammation resolution and play an atheroprotective role ${ }^{56,57}$. In LPS-stimulated cardiomyocytes, overexpression of CTRP12 blocks the generation and release 


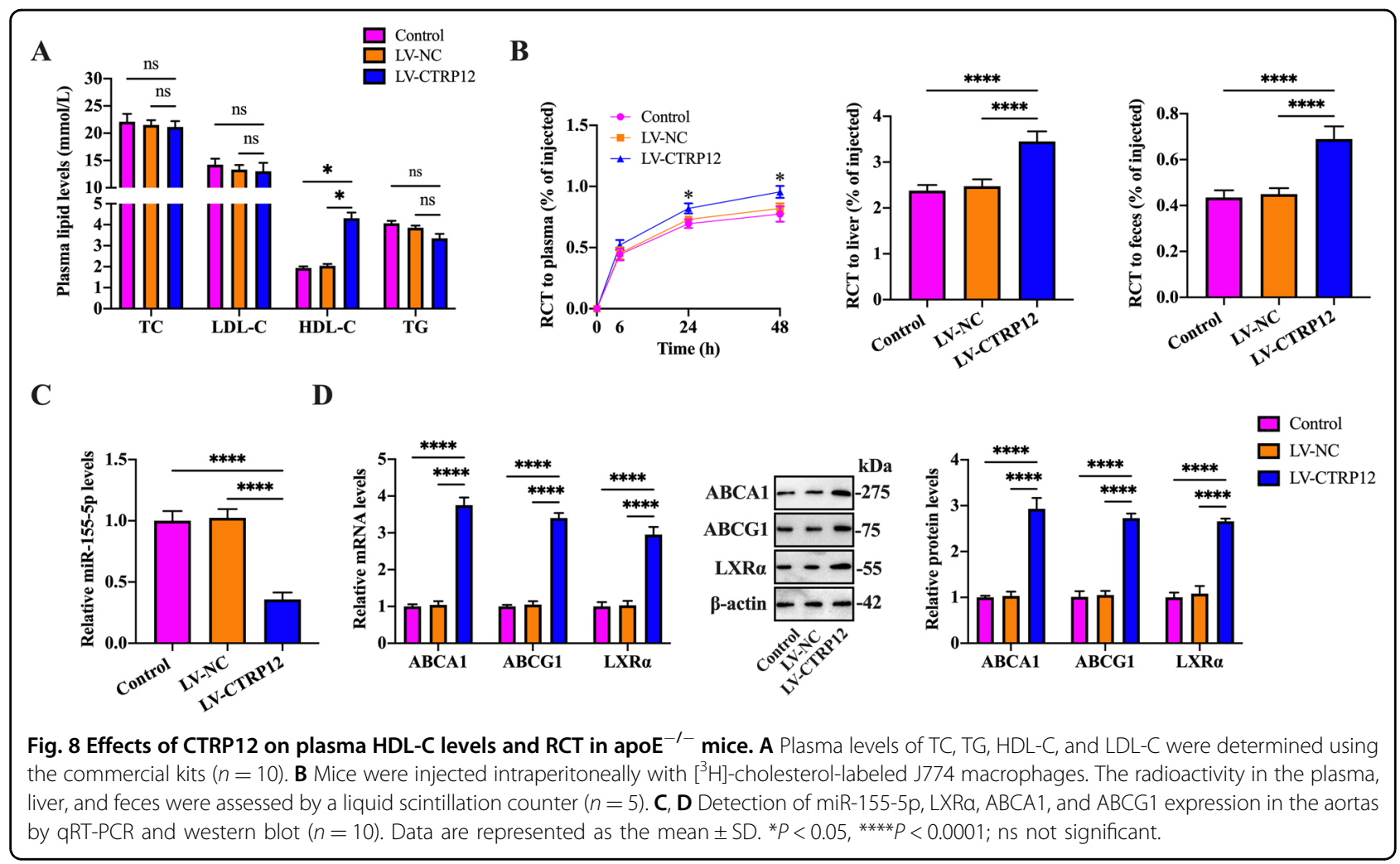

of pro-inflammatory cytokines ${ }^{58}$. Treatment with recombinant CTRP12 decreases the expression of MCP-1, TNF$\alpha$ and IL- 6 in LPS-treated macrophages ${ }^{59}$. Injection of miR-155-5p agomir increases TNF- $\alpha$, IL- $1 \beta$, and IL-6 amounts in the ventral midbrain of Parkinson's disease mice ${ }^{60}$. Additionally, LXR $\alpha$ overexpression induces M1 to M2 phenotypic transition in macrophages ${ }^{61}$. Our results showed that CTRP12 polarized macrophages towards an M2 phenotype and attenuated vascular inflammation through the miR-155-5p/LXR $\alpha$ pathway, thereby providing another important mechanism for its antiatherogenic action. A growing body of evidence has indicated that lncRNAs are pivotal regulators of atherogenesis ${ }^{62,63}$. More recently, Wang et al. reported that the expression levels of CTBP1-AS2, a lncRNA, were significantly decreased in the serum of patients with atherosclerosis, and its overexpression inhibits proliferation and promotes autophagy in human aortic smooth muscle cells challenged with ox$\mathrm{LDL}^{64}$. Another study revealed that CTBP1-AS2 sponges miR-155-5p to suppress pro-inflammatory cytokine production induced by high glucose in human glomerular mesangial cells ${ }^{65}$. Thus, it is possible that CTBP1-AS2 is involved in CTRP12-induced downregulation of miR-155$5 \mathrm{p}$ expression. Future studies will be needed to confirm this possibility.

In summary, the present study has demonstrated a novel role of macrophage CTRP12 in inhibiting the development of atherosclerosis and uncovered a novel mechanism underlying the regulation of $\mathrm{ABCA} 1$ and ABCG1. CTRP12 decreases miR-155-5p levels and then increases LXR $\alpha$ expression, which promotes ABCA1- and ABCG1-dependent cholesterol efflux and alleviates inflammatory response. These data extend our understanding for the biological functions of CTRP12 and provide a potential therapeutic target for atherosclerotic cardiovascular disease.

\section{Acknowledgements}

We thank Dr Zhen-Wang Zhao and Institute of Cardiovascular Research, Key Laboratory for Atherosclerology of Hunan Province, University of South China for technical support.

\section{Funding}

This work was financially supported by Scientific Research Program of Higher Education of Hainan province (Hnky2020-43), Scientific Research of BSKY from Anhui Medical University (XJ201803), Natural Science Foundation of Anhui Higher Education Institutions (KJ2019A0225), and Hainan Provincial Natural Science Foundation of China (820RC767).

\section{Author details}

'Department of Cardiology, The First Affiliated Hospital of University of South China, Hengyang 421001 Hunan, China. ${ }^{2}$ Institute of Clinical Medicine, The Second Affiliated Hospital of Hainan Medical University, Haikou 570100 Hainan, China. ${ }^{3}$ Department of Pathophysiology, School of Basic Medical Sciences,

Anhui Medical University, Hefei 230032 Anhui, China. ${ }^{4}$ Department of Neonatology, The First Affiliated Hospital of University of South China, Hengyang 421001 Hunan, China 


\section{Author contributions}

X.-H.Y. and S.-H.Y. conceived of the presented idea. G.W. and J.-J.C. designed and performed the experiments. K.R. and W.-Y.D. analyzed the experimental data. All authors discussed the results and contributed to the final manuscript.

\section{Conflict of interest}

The authors declare no competing interests.

\section{Ethics statement}

This study was approved by the Ethics Committees of The Second Affiliated Hospital of Hainan Medical University.

\section{Publisher's note}

Springer Nature remains neutral with regard to jurisdictional claims in published maps and institutional affiliations.

Supplementary information The online version contains supplementary material available at https://doi.org/10.1038/s41419-021-03544-8.

Received: 14 November 2020 Revised: 18 February 2021 Accepted: 19 February 2021

Published online: 10 March 2021

\section{References}

1. Lacy, M. et al. Interactions between dyslipidemia and the immune system and their relevance as putative therapeutic targets in atherosclerosis. Pharmacol. Ther. 193, 50-62 (2019).

2. Yuan, Y., Li, P. \& Ye, J. Lipid homeostasis and the formation of macrophagederived foam cells in atherosclerosis. Protein Cell 3, 173-181 (2012).

3. Westerterp, M. et al. ATP-binding cassette transporters, atherosclerosis, and inflammation. Circulation Res. 114, 157-170 (2014).

4. Yvan-Charvet, L., Wang, N. \& Tall, A. R. Role of HDL, ABCA1, and ABCG1 transporters in cholesterol efflux and immune responses. Arterioscler Thromb. Vasc. Biol. 30, 139-143 (2010).

5. He, Y. et al. RIP140 triggers foam-cell formation by repressing ABCA1/G1 expression and cholesterol efflux via liver $X$ receptor. FEBS Lett. 589, 455-460 (2015).

6. Wang, G. et al. Fargesin alleviates atherosclerosis by promoting reverse cholesterol transport and reducing inflammatory response. Biochim. et. Biophys. Acta Mol. Cell Biol. Lipids 1865, 158633 (2020).

7. Wang, H. et al. Sonodynamic therapy-induced foam cells apoptosis activates the phagocytic PPARY-LXRa-ABCA1/ABCG1 pathway and promotes cholesterol efflux in advanced plaque. Theranostics 8, 4969-4984 (2018).

8. Bäck, M., Yurdagul, A. Jr., Tabas, I., Öörni, K. \& Kovanen, P. T. Inflammation and its resolution in atherosclerosis: mediators and therapeutic opportunities. Nat. Rev. Cardiol. 16, 389-406 (2019).

9. Rigamonti, E. et al. Liver $X$ receptor activation controls intracellular cholesterol trafficking and esterification in human macrophages. Circulation Res. 97, 682-689 (2005).

10. Jiang, T. et al. Leonurine prevents atherosclerosis via promoting the expression of $A B C A 1$ and $A B C G 1$ in a Ppary/Lxra signaling pathway-dependent manner. Cell. Physiol. Biochem. 43, 1703-1717 (2017).

11. Liu, X. et al. Kuwanon $\mathrm{G}$ attenuates atherosclerosis by upregulation of $\mathrm{LXRa}$ ABCA1/ABCG1 and inhibition of NFKB activity in macrophages. Toxicol. Appl. Pharmacol. 341, 56-63 (2018).

12. Zeng, Y. et al. Dihydromyricetin ameliorates foam cell formation via LXRaABCA1/ABCG1-dependent cholesterol efflux in macrophages. Biomed. Pharmacother. 101, 543-552 (2018).

13. Zhao, $L$. et al. The roles of liver $X$ receptor $a$ in inflammation and inflammation-associated diseases. J. Cell Physiol. 2020. https:/doi.org/10.1002/ jcp.30204. [ahead of print].

14. Feinberg, M. \& Moore, K. MicroRNA regulation of atherosclerosis. Circulation Res. 118, 703-720 (2016).

15. Schober, A. \& Weber, C. Mechanisms of MicroRNAs in atherosclerosis. Annu. Rev. Pathol. 11, 583-616 (2016)

16. Faraoni, I., Antonetti, F., Cardone, J. \& Bonmassar, E. miR-155 gene: a typical multifunctional microRNA. Biochim. et. Biophys. Acta 1792, 497-505 (2009).
17. Ormseth, M. et al. Utility of select plasma MicroRNA for disease and cardiovascular risk assessment in patients with rheumatoid arthritis. J. Rheumatol. 42, 1746-1751 (2015).

18. Singh, S. et al. MiR-223-3p and miR-122-5p as circulating biomarkers for plaque instability. Open Heart 7, e001223, https://doi.org/10.1136/openhrt-2019001223 (2020). [ahead of print].

19. Barberio, M. et al. Cholesterol efflux alterations in adolescent obesity: role of adipose-derived extracellular vesical microRNAs. J. Transl. Med. 17, 232 (2019).

20. Li, H. F. et al. Inhibition of miR-155 potentially protects against lipopolysaccharide-induced acute lung injury through the IRF2BP2-NFAT1 pathway. Am. J. Physiol. Cell Physiol. 319, C1070-c81 (2020).

21. Wei, Z. et al. C1q/TNF-related protein-12 (CTRP12), a novel adipokine that improves insulin sensitivity and glycemic control in mouse models of obesity and diabetes. J. Biol. Chem. 287, 10301 (2012).

22. Zhou, M. et al. CTRP12 ameliorated lipopolysaccharide-induced cardiomyocyte injury. Chem. Pharm. Bull. 68, 133-139 (2020).

23. Ogawa, $\mathrm{H}$. et al. Adipolin/CTRP12 protects against pathological vascular remodelling through suppression of smooth muscle cell growth and macrophage inflammatory response. Cardiovascular Res. 116, 237-249 (2020).

24. Fadaei, R. et al. Decreased serum levels of CTRP12/adipolin in patients with coronary artery disease in relation to inflammatory cytokines and insulin resistance. Cytokine 113, 326-331 (2019).

25. Li, Z. et al. Krüppel-Like factor 4 regulation of cholesterol-25-hydroxylase and liver $X$ receptor mitigates atherosclerosis susceptibility. Circulation 136 1315-1330 (2017)

26. Zhao, Z. W. et al. Heat shock protein 70 accelerates atherosclerosis by downregulating the expression of ABCA1 and ABCG1 through the JNK/Elk-1 pathway. Biochim Biophys. Acta Mol. Cell Biol. Lipids 1863, 806-822 (2018).

27. Xia, $X$. et al. Myocardin suppression increases lipid retention and atherosclerosis via downregulation of ABCA1 in vascular smooth muscle cells. Biochim. et Biophys. Acta Mol. Cell Biol. Lipids 1866, 158824 (2020).

28. Shashkin, P., Dragulev, B. \& Ley, K. Macrophage differentiation to foam cells, Curr. Pharm. Des. 11, 3061-3072 (2005).

29. Chen, W., Silver, D. L., Smith, J. D. \& Tall, A. R. Scavenger receptor-Bl inhibits ATP-binding cassette transporter 1- mediated cholesterol efflux in macrophages. J. Biol. Chem. 275, 30794-30800 (2000).

30. Haidar, B., Denis, M., Krimbou, L., Marcil, M. \& Genest, J. Jr. CAMP induces ABCA1 phosphorylation activity and promotes cholesterol efflux from fibroblasts. J. Lipid Res 43, 2087-2094 (2002).

31. Chistiakov, D. A., Melnichenko, A. A., Myasoedova, V. A.r Grechko, A. V. \& Orekhov, A. N. Mechanisms of foam cell formation in atherosclerosis. J. Mol. Med. 95, 1153-1165 (2017).

32. Wang, $\mathrm{X}$. et al. Adiponectin improves NF-KB-mediated inflammation and abates atherosclerosis progression in apolipoprotein E-deficient mice. Lipids Health Dis. 15, 33 (2016).

33. Okamoto, Y. et al. Adiponectin reduces atherosclerosis in apolipoprotein Edeficient mice. Circulation 106, 2767-2770 (2002).

34. Gordon, D. J. et al. High-density lipoprotein cholesterol and cardiovascular disease. Four prospective American studies. Circulation 79, 8-15 (1989).

35. Ren, K. et al. Mangiferin promotes macrophage cholesterol efflux and protects against atherosclerosis by augmenting the expression of $\mathrm{ABCA} 1$ and $\mathrm{ABCG}$. Aging 11, 10992-11009 (2019).

36. Tan, S. et al. CTRP12 ablation differentially affects energy expenditure, body weight, and insulin sensitivity in male and female mice. Am. J. Physiol. Endocrinol. Metab. 319, E146-E162 (2020).

37. Enomoto, T. et al. Adipolin/C1qdc2/CTRP12 protein functions as an adipokine that improves glucose metabolism. J. Biol. Chem. 286, 34552-34558 (2011).

38. Tan, S. et al. Partial deficiency of CTRP12 alters hepatic lipid metabolism. Physiological genomics 48, 936-949 (2016).

39. Sargolzaei, J., Chamani, E., Kazemi, T., Fallah, S. \& Soori, H. The role of adiponectin and adipolin as anti-inflammatory adipokines in the formation of macrophage foam cells and their association with cardiovascular diseases. Clin. Biochem. 54, 1-10 (2018).

40. Tian, L. et al. Adiponectin reduces lipid accumulation in macrophage foam cells. Atherosclerosis 202, 152-161 (2009).

41. Tian, L et al. Adiponectin-AdipoR1/2-APPL 1 signaling axis suppresses human foam cell formation: differential ability of AdipoR1 and AdipoR2 to regulate inflammatory cytokine responses. Atherosclerosis 221, 66-75 (2012).

42. Tan, S. Y., Little, H. C., Sarver, D. C., Watkins, P. A. \& Wong, G. W. CTRP12 inhibits triglyceride synthesis and export in hepatocytes by suppressing HNF-4a and DGAT2 expression. FEBS Lett. 594, 3227-3239 (2020). 
43. Adorni, M. et al. The roles of different pathways in the release of cholesterol from macrophages. J. Lipid Res. 48, 2453-2462 (2007).

44. Peng, Y., Xu, J., Zeng, Y., Chen, L. \& Xu, X. Polydatin attenuates atherosclerosis in apolipoprotein E-deficient mice: role of reverse cholesterol transport. Phytomedicine 62, 152935 (2019).

45. de Villiers, W. \& Smart, E. Macrophage scavenger receptors and foam cell formation. J. Leukoc. Biol. 66, 740-746 (1999).

46. Shen, D. et al. Geniposide against atherosclerosis by inhibiting the formation of foam cell and lowering reverse lipid transport via p38/MAPK signaling pathways. Eur. J. Pharmacol. 864, 172728 (2019).

47. Wang, Z. et al. Naoxintong retards atherosclerosis by inhibiting foam cell formation through activating Ppara pathway. Curr. Mol. Med. 18, 698-710 (2018).

48. Lin, $\mathrm{H}$. et al. Andrographolide inhibits oxidized LDL-induced cholesterol accumulation and foam cell formation in macrophages. Am. J. Chin. Med. $\mathbf{4 6}$ 87-106 (2018)

49. Schmitz, G. \& Langmann, T. Transcriptional regulatory networks in lipid metabolism control ABCA1 expression. Biochim. et. Biophys. Acta 1735, 1-19 (2005).

50. Ou, X. et al. Angiopoietin-1 aggravates atherosclerosis by inhibiting cholesterol efflux and promoting inflammatory response. Biochim. et. Biophys. Acta Mol. Cell Biol. Lipids 1865, 158535 (2020).

51. Tang, S. et al. Pregnancy-associated plasma protein-A accelerates atherosclerosis by regulating reverse cholesterol transport and inflammation. Circulation J. 83, 515-523 (2019).

52. Jin, P. et al. Homocysteine accelerates atherosclerosis via inhibiting LXRamediated ABCA1/ABCG1-dependent cholesterol efflux from macrophages. Life Sci. 214, 41-50 (2018).

53. Gaudet, A. et al. miR-155 deletion in female mice prevents diet-induced obesity. Sci. Rep. 6, 22862 (2016).

54. Zhu, M. et al. MafbHyperlipidemia-Induced MicroRNA-155-5p Improves $\beta$-Cell Function by Targeting. Diabetes 66, 3072-3084 (2017).
55. Chen, L. et al. MALAT1 overexpression attenuates AS by inhibiting ox-LDLstimulated dendritic cell maturation via miR-155-5p/NFIA axis. Cell Cycle 19 2472-2485 (2020)

56. Barrett, T. J. Macrophages in atherosclerosis regression. Arterioscler Thromb. Vasc. Biol. 40, 20-33 (2020).

57. Yang, S. et al. Macrophage polarization in atherosclerosis. Clin. Chim. Acta 501, 142-146 (2020)

58. Zhou, M. Q. et al. CTRP12 ameliorated lipopolysaccharide-induced cardiomyocyte injury. Chem. Pharm. Bull. 68, 133-139 (2020).

59. Ogawa, $\mathrm{H}$. et al. Adipolin/CTRP12 protects against pathological vascular remodelling through suppression of smooth muscle cell growth and macrophage inflammatory response. Cardiovasc. Res. 116, 237-249 (2020).

60. LV, R. et al. Rosmarinic acid alleviates inflammation, apoptosis, and oxidative stress through regulating miR-155-5p in a mice model of Parkinson's disease. ACS Chem. Neurosci. 11, 3259-3266 (2020).

61. Liu, M. et al. LXRa is expressed at higher levels in healthy people compared to atherosclerosis patients and its over-expression polarizes macrophages towards an anti-inflammatory MФ2 phenotype. Clin. Exp. Hypertens. 40, 213-217 (2018)

62. $Y u, X . H$. et al. LncRNA kcnq1ot1 promotes lipid accumulation and accelerates atherosclerosis via functioning as a ceRNA through the miR-452-3p/HDAC3/ ABCA1 axis. Cell Death Dis. 11, 1043 (2020).

63. Simion, $V$. et al. A macrophage-specific IncRNA regulates apoptosis and atherosclerosis by tethering HuR in the nucleus. Nat. Commun. 11, 6135 (2020).

64. Wang, Y., Zhang, C. X., Ge, S. L. \& Gong, W. H. CTBP1-AS2 inhibits proliferation and induces autophagy in ox-LDL-stimulated vascular smooth muscle cells by regulating miR-195-5p/ATG14. Int J. Mol. Med. 46, 839-848 (2020).

65. Wang, G., Wu, B., Zhang, B., Wang, K. \& Wang, H. LncRNA CTBP1-AS2 alleviates high glucose-induced oxidative stress, ECM accumulation, and inflammation in diabetic nephropathy via miR-155-5p/FOXO1 axis. Biochem. Biophys. Res. Commun. 532, 308-314 (2020) 